\title{
Effect of Laser Surface Texturing (LST) on Tribochemical Films Dynamics and Friction and Wear Performance*
}

\author{
Bolutife Olofinjana ${ }^{1,2}$, Cinta Lorenzo-Martin ${ }^{1}$, Oyelayo O. Ajayi ${ }^{1}$, and Ezekiel O. Ajayi ${ }^{2}$ \\ ${ }^{1}$ Energy Systems Division \\ Argonne National Laboratory \\ Argonne, IL 60439 \\ ${ }^{2}$ Department of Physics \\ Obafemi Awolowo University \\ Ile-Ife, Nigeria \\ Phone (630) 252-9021 \\ Fax (630) 252-4798 \\ ajayi@anl.gov
}

\section{Abstract:}

Surface texturing or topographical design is one of the primary techniques to control friction and wear performance of surfaces in tribological contact. Laser surface texturing (LST), whereby a laser beam is used to produce regular arrays of dimples on a surface, has been demonstrated to reduce friction in conformal lubricated contacts. Friction and wear behavior under boundary lubrication is also known to be dependent on the formation and durability of the tribochemical film formed from lubricant additives. In this paper, the effects of LST on the formation and durability of tribochemical films and its consequent impacts on friction and wear behavior in various lubrication regimes were evaluated. Friction and wear tests that cycled through different lubrication regimes were conducted with both polished and LST treated surfaces using a synthetic lubricant with and without model additives of ZDDP and MoDTC mixture. In the base oil without additives, LST produced noticeable reduction in friction in all lubrication regimes. However, with low-friction model additives, friction was higher in tests with LST due to significant differences in the tribochemical film formation in the polished and LST surfaces, as well as the sliding counterface. Continuous tribo-films were formed on ball conterface rubbed against polished surfaces while the films were streaky and discontinuous in ball rubbed against LST surfaces. LST produced more wear on the ball

\footnotetext{
* Work supported by the Department of Energy, under Contract DE-AC02-06CH11357. 
counterface in both base and additized oils. No measurable wear was observed in both the polished and LST flat specimens.

\section{Introduction}

Sustainable friction and wear control in components whose surfaces are in contact and relative motion is essential for efficiency, reliability and durability of such components. Although, there may be components and systems in which relatively high friction is desirable (for instance break systems), friction reduction is often the goal in most components and systems. Similarly, wear reduction is also highly desirable in most tribological components.

Surface texturing, in its many different forms, is one of the common approaches that has been utilized for friction and wear control on component surfaces for a long time. Surface texturing essentially involves the creation and insertion of some regular topographical features of different forms, sizes and shapes into an otherwise plain surface. An example of surface texturing currently in use by industries, that is perhaps most familiar is the cross hatch honing of cylindrical liner of internal combustion engine (ICE), whereby grooves that are intersecting at same angles are created on the liner surface. The presences of these grooves enhances effective lubrication of the piston ring and cylinder liner interface during engine operation. Another example that may be familiar involves some surface mechanical treatments, such as shot peening, that leave topographical features on the surface. Such features can indeed be considered a form of texturing. 
In recent years, texturing of tribological surfaces is used primarily to describe surface topographical modification consisting of some patterned micro dimples on bearing surfaces. These dimples have been produced by a variety of techniques including mechanical, e.g. [1], lithographic etching, e.g. [2,3], etc. Over the last decade or so, one of the most commonly used technique for surface texturing with dimples is by laser $[4,5]$. The process of laser surface texturing (LST) involves creation of micro-dimples via a material ablation mechanism. These dimples are assumed to function as traps for wear debris, serve as reservoir for lubricant under starved lubrication condition and possibly enhance hydrodynamic lubrication mechanism.

Numerous analytical and experimental studies have been conducted on the impact of LST on tribological performance of lubricated sliding contacts focusing primarily on fluid film lubrication [e.g., 4-10]. Results of these studies showed that in conformal contacts and unidirectional sliding, such as mechanical seals and journal bearings, LST can enhance tribological performance in terms of friction reduction and increase in load carrying capacity. For such contract configuration, the improved tribological performance is attributed to LST shifting the transition between the boundary and mixed lubrication regimes to a lower value of Sommerfeld number in addition to reducing the friction under boundary lubrication regime [6]. However, for conformal contact under reciprocating sliding in 2 to $10 \mathrm{~Hz}$ frequency range, LST was observed to be detrimental by increasing friction under oil lubrication [7]. In non-conformal contact configuration, LST has been shown to reduce the lubricant fluid film thickness downstream of the contact area [9]. Such a reduction in lubricant fluid film thickness can lead to accelerated wear, as was observed in a study with a ball in unidirectional sliding against LST treated flat [10]. 
After the initial wear and the eventual change in contact geometry to a conformal configuration, significant friction reduction can indeed be observed with LST treated surfaces starting initially with a non-conformal contact.

Under boundary lubrication regime, extensive interaction occurs between the surfaces in sliding contact, eventually resulting in the formation of tribochemical surface films primarily from the additives in the oil. The films are usually formed, at least initially, at the real areas of contact between the sliding surfaces. Consequently, the location and extent of the tribofilm formation is strongly dependent on the surface roughness and surface texture which determines the real area of contact. The formation and the nature of the tribochemical film is a major determinant in the friction and wear behavior of surfaces in contact under boundary lubrication regime [11]. The various functional additions in lubricants are usually main participants in the formation of tribochemical films.

There are different classes of lubricant additives with different tribological functional attributes. These include anti-wear (to reduce wear), friction modifiers (to reduce friction) and extreme pressure (to increase scuffing resistance). Some additives function through molecular adsorption onto the sliding surfaces, other function through chemical reaction to form a new layer on the surface, i.e., the tribochemical films. In the present study, additives that form durable (wear resistant) surface layer with low-friction behavior will be investigated. Several studies have shown that a mixture of an anti-wear (Zinc dialkyldithiophosphate - ZDDP) and an organometallic friction modifier (Molybdenum diothiocarbonate - MoDTC] additives when incorporated into a synthetic 
base oil PAO do indeed produce a durable low-friction tribochemical surface films under boundary lubrication regime [e.g., 12-14]. Hence, the current study will use this additive combination to illustrate the LST and lubricant chemistry interactions during sliding contact.

The main objective of the present study is the exploration of simultaneous friction reduction in hydrodynamic as well as the boundary lubrication regimes by combining two approaches that have been shown to respectively reduced friction in these regimes. This paper presents an experimental investigation of combining LST, which has been shown to be capable of reducing friction under fluid film lubrication regime, with oil additives that have been shown to produce low-friction tribochemical films under the boundary lubrication regime. Conceivably, such a combination can lead to friction reduction across all lubrication regimes. The results of the study can indeed have an implication for combining various (materials, surface, lubricant) technologies to achieve desired tribological performance goals.

\section{Experimental Details:}

Friction and wear tests were conducted using a ball-on-flat contact configuration in a unidirectional sliding with a commercially available pin-on-disc test rig (NANOVEA). The ball specimen is made of a $12.7 \mathrm{~mm}(1 / 2$ ”) diameter AISI 52100 bearing ball with a hardness of $62 R_{c}$ and surface roughness of $0.03 \mu \mathrm{m} \mathrm{R}$. The flat specimen is made of 50 x 40 x $7.5 \mathrm{~mm}$ rectangular disc of hardened H-13 tool steel with a nominal hardness of about $61 \mathrm{R}_{\mathrm{c}}$. The disc specimen were finished in two ways; polished and LST followed by polishing. The polished flat has a final surface finished of $0.05 \mu \mathrm{m} \mathrm{Ra}$. LST 
treatment consists of array of dimples with an average diameter of $100 \mu \mathrm{m}$, average depth of $10 \mu \mathrm{m}$ and center-to-center distance between dimples of about $240 \mu \mathrm{m}$. The surface finish of area between dimples is about $0.04 \mu \mathrm{m} \mathrm{Ra}$. This particular LST pattern was observed to the most effective in terms of friction reduction in conformal unidirectional sliding [18]. It should be noted that the LST flat surface in general is isotropic and hence minimal variation is expected in friction as the ball contact travels the circular path during testing. The number of dimples in the contact patch may vary with time; this could make the friction somewhat noisy. The type frictional anisotropy observed in tests using flat specimens with a grinding lay is not expected with the LST surface [19]. Figure 1 shows the optical micrograph and profilometry of the surfaces of the polished and LST flat specimens.

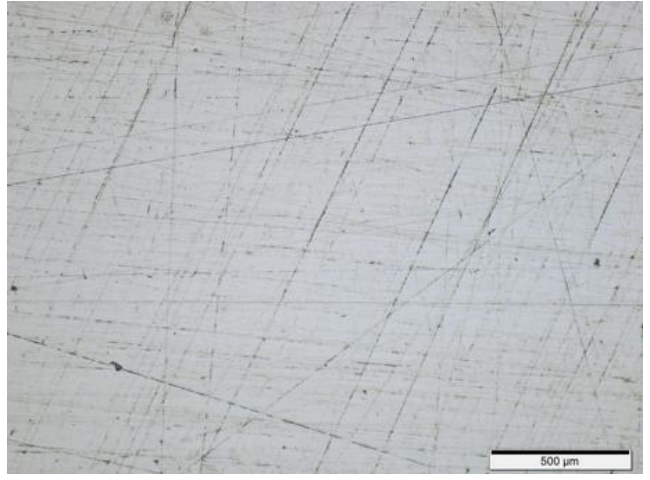

(a)

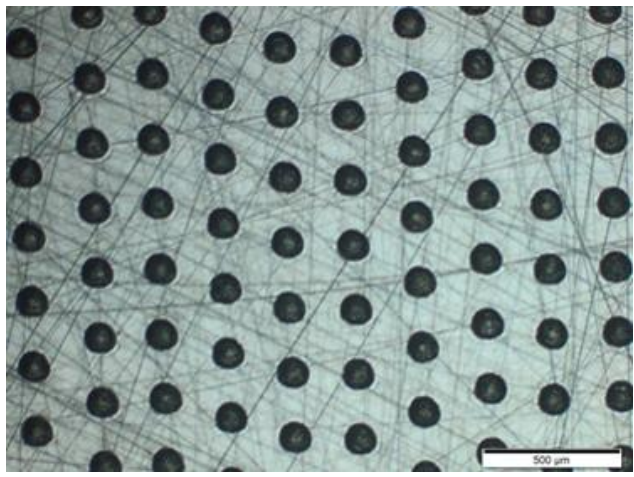

(b)

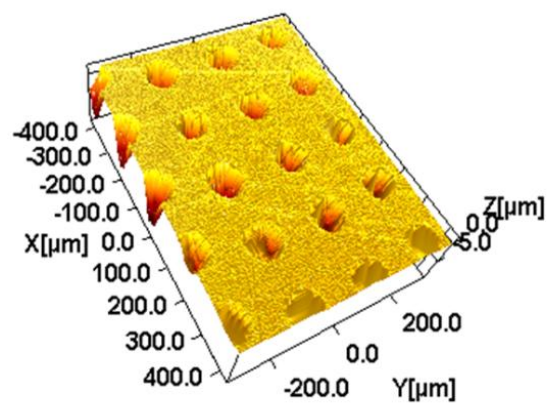

(c) 
Figure 1: Optical Micrograph of (a) polished and (b) LST flat specimens. (c) Optical profilometry of LST flat specimen

Friction and wear tests were conducted at a constant normal load of $5 \mathrm{~N}$ (maximum contact pressure of $0.7 \mathrm{GPa}$ ) and a constant temperature of $75^{\circ} \mathrm{C}$. A protocol consisting of three cycles of discrete speed varying was used to move the lubricated contact of the test through different lubrication regimes in a reversible manner. Each test started with a speed of $200 \mathrm{rpm}$ for 2 minutes; followed by the following sequence of discrete step speed (rpm) decrease every 2 minutes 150, 100, 80, 60, 40, 30, 25, 20, 15, 10, 9, 8, 7, 6, $5,4,3,2,1,0.8,0.6,0.4$. For a track diameter of $11 \mathrm{~mm}$ used in the test, the varying speed protocol translates to a linear speed range of 0.288 -to- $0.0005 \mathrm{~m} / \mathrm{s}$. This is first stage of Cycle 1. This is followed by a discrete step increase in a reverse order and for the same duration; this is second stage of Cycle 1. Two more cycles, same as Cycle 1, followed as shown in Figure 2. The total duration of each test consisting of the three speed cycles was about 320 minutes. Although, the speed variation protocol used in this study did not simulate any particular component or application, it provides a more realistic assessment of tribological mechanisms in real components when compared to the usual and most common constant speed tests. In most components and systems, there are starts and stops as well as variable speeds during normal operation.

Tests were conducted with two lubricants; a polyalphaolefin synthetic base oil (PAO4) with $18 \mathrm{cSt}$ viscosity at $40^{\circ} \mathrm{C}$ and $4 \mathrm{cSt}$ at $100^{\circ} \mathrm{C}$. The second lubricant is PAO4 +2.5 ZDDP + 2.5 MoDTC, designated PAO4 with additives. Viscosity measurement showed 
that incorporation of the additives did not change the lubricant viscosity. All the tests were conducted under fully flooded condition.

During each test, the friction coefficient was continuously monitored and the lubricant temperature was maintained at $75^{\circ} \mathrm{C}$. At the conclusion of the tests, wear on both the ball and flat specimens were assessed using optical profilometry. The worn surfaces were also examined by microscopy to assess the wear mechanisms.

\section{Results and Discussions:}

The variation of measured friction coefficient with time during the entire test duration and different speed profile for the three cycles is shown in Figure 2. In the tests with PAO4 base fluid, the trend in the friction behavior with polished and textured flats are similar to one another, but with different magnitude. The magnitude of friction in tests with textured surface was always lower than those in the test with polished surface under the same condition. In both cases, there was a gradual slight increase during the first 3 or 4 speed decrease. This initial increase in friction is attributed to decrease of lubricant fluid film thickness as the speed decreases and the consequent increased interaction between surfaces. Over the next 9-10 step decrease, the friction was nearly constant until the very low speed of $5 \mathrm{rpm}$ when the friction suddenly increased and became very noisy. This is indicative of severe boundary lubrication regime is which a stick-slip phenomenon is a common occurrence $[15,16]$. Sometimes, noisy friction behavior could be an indication of occurrence of galling. However, in the present study, there were no surface damage features indicative of galling. Indeed, there was minimal damage, if any, on both the polished and LST flat specimens. During the speed increase stage 2 of the first cycle, the friction coefficient decreased almost linearly. This is in part the result of 
gradual increase in fluid film thickness and its contribution to friction as the speed increases. Change in contact geometry from point non-conformal contact to a more conformal contact is also expected as wear occurs on the ball during the speed reduction step of the first cycle. Furthermore, run-in process during the speed decrease stage is expected to contribute to differences in friction during the speed decrease and the speed increase stages of the first cycle. Yet, another plausible reason is the variation in nominal contact pressure as wear occurs during speed decrease stage 1 of first cycle. For non-conformal contact configuration, as in the present study, it has been shown that the nominal contact pressure decreases exponentially with time as wear occurs [20]. Such a significant in contact pressure will have a noticeable effect on the tribological phenomena at the contact interface which can be reflected in the friction behavior.

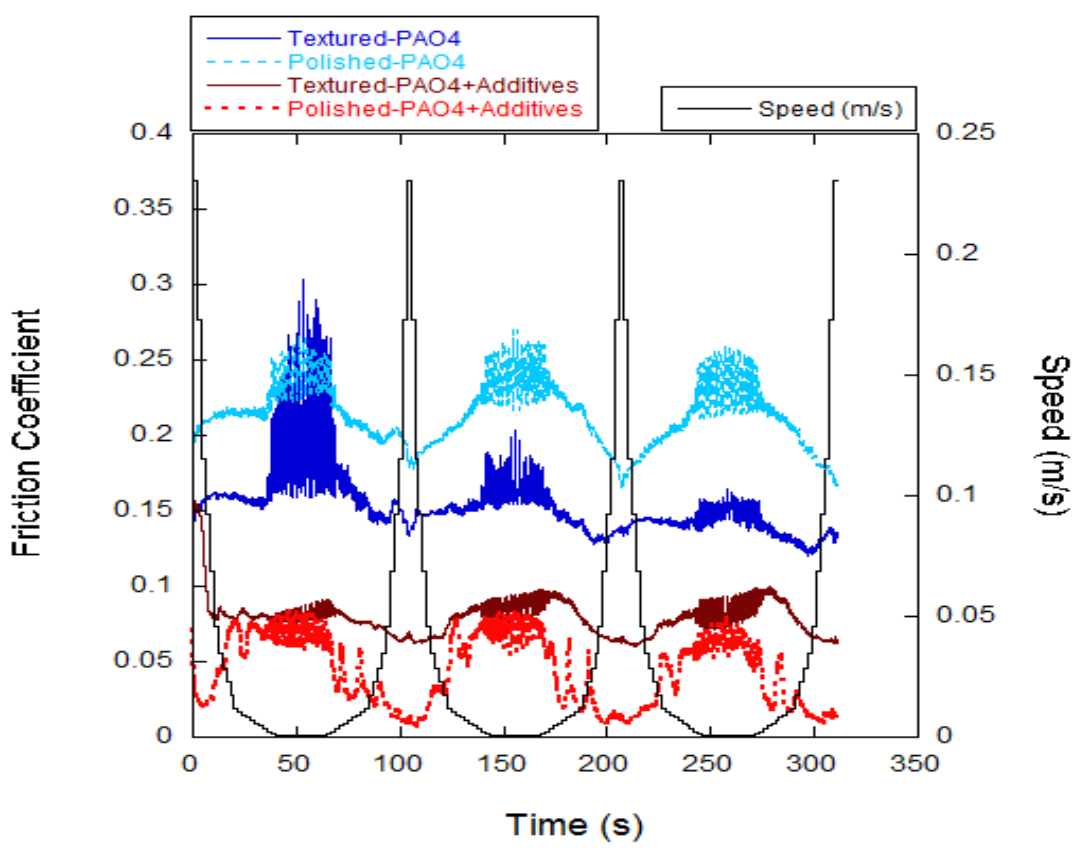

Figure 2: Variation of friction coefficient and sliding speed with time showing the three speed cycles 
Friction variation during Cycle 2 and 3 reflects some of these changes at the contact interface during Cycle 1. For the test with polished flat, the friction increased or decreased nearly linearly during the respective speed decrease and increase, except at very low-speed with extreme boundary regime with its stick-slip phenomenon. The amplitude of the friction noise in this regime is reduced during Cycle 2 and 3 . In the test with the textured flat, the variation of friction with speed was much less than for polished specimen. The extreme boundary regime noise is still noticeable, but substantially truncated in subsequent cycles. These results are consistent with earlier observations that LST accelerate the run-in of lubricated surfaces and expands the range of contact condition for the operation of fluid film lubrication regime $[6,10]$.

The frictional behaviors in tests with lubricant containing ZDDP+MoDTC additives were different as indicated on Figure 2. In general, the friction coefficients in tests with both polished and textured flat were lower compared to tests with basestock PAO4. This is due to the formation of low-friction tribochemical films from the additives. Significant differences were also observed in the friction behavior in tests with polished and textured flat. For the polished flat, the friction coefficient decrease quickly during initial speed reduction as the low-friction tribochemical films were being formed. As the fluid film decreased further with more reduction in speed, the contact moves more into boundary regime and friction began to show more variation as the tribofilms are being and removed. In the test with textured surface the frictional behavior was similar to the one for the base oil, but with a much less value. The stick-slip like behavior under severe boundary regime was also observed. The overall friction coefficient was higher in the 
test with textured surface compared to the polished flat; note the opposite is true for test with base fluid.

The combined impact of LST and presence of additives on friction behavior of lubricated contact in various lubrication regimes is best illustrated by Figure 3. The figure is a plot of friction coefficient as a function of the calculated $\lambda$ ratio, i.e., the ratio of lubricant fluid film thickness $(\mathrm{h})$ and the composite surface roughness $(\sigma)$ of the two surfaces in contact at various sliding speeds. Figure 3 is essentially a Stribeck curve with the $\lambda$ ratio on the $x$-axis indicating the operating lubrication regime. The $\lambda$ ratio was calculated for each discrete speed during the three speed variation cycles. Lines 1 and 2 are for the first cycle, 3 and 4 for $2^{\text {nd }}$ cycle and 5, 6 for the $3^{\text {rd }}$ cycles correspondingly. The odd numbers are for speed decrease stage of the cycle, while the even numbers are for speed increase stage. In the tests with base fluid, the friction coefficient is nearly constant for textured surfaces until the very low $\lambda$ ratio (Fig. 3a). Similar trends were observed for test with polished disc but with higher magnitude for all $\lambda$ ratio. The operating lubrication regime can be estimated from the value of $\lambda$ ratio. For $\lambda$ ratio greater than 1 , the surfaces are completely separated by fluid film, and the shear of lubricant fluid film determines the friction behavior. As $\lambda$ value decreases, interactions between surfaces also contribute to the friction. At extremely low $\lambda$ (severe boundary) shear of the asperities in contact becomes a major contributor to friction. In the extreme case, uneven plastic flow of asperities in contact can produce noisy friction as was observed in the present study. 


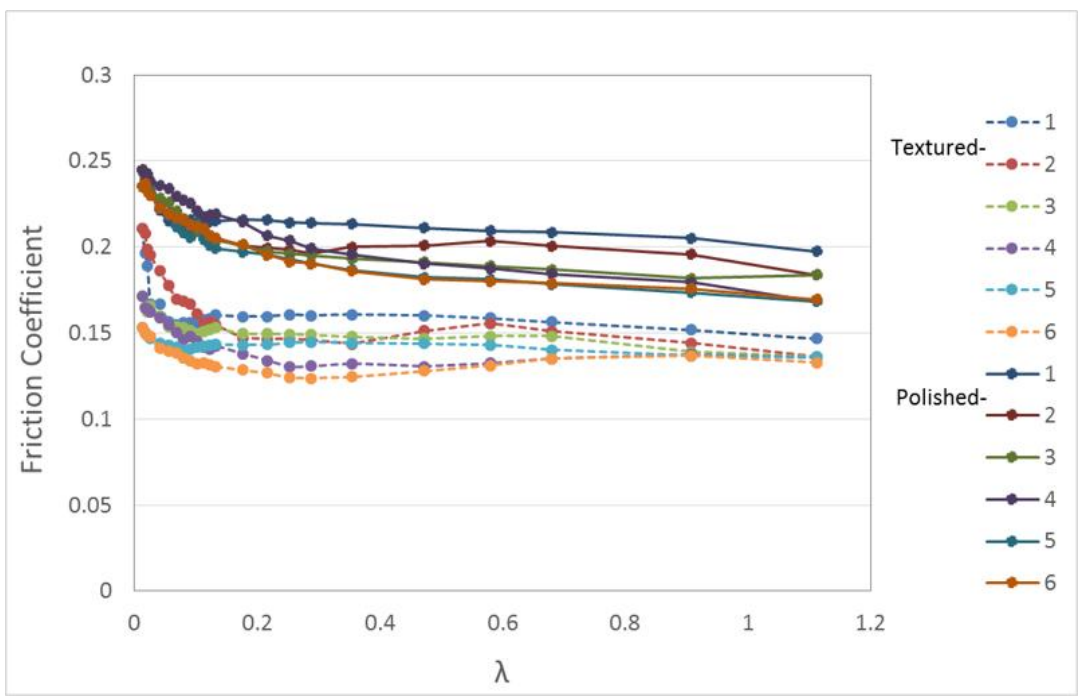

(a)

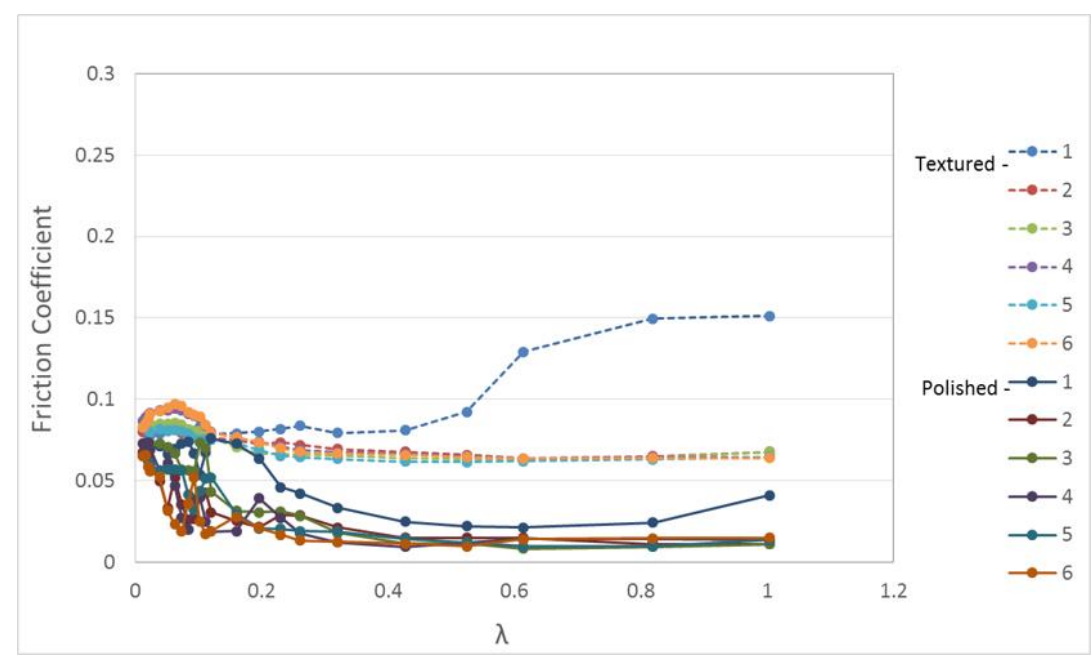

(b)

Figure 3: Variation of friction with $\lambda$ ratio for (a) base PAO oil and (b) additized oil comparing polished LST surfaces.

When additives were added to the lubricant to produce tribochemical surface film, striking differences can be seen in the friction behavior between polished and textured disc (Fig. 3b). For the polished disc, the friction was much lower than for textured test. In both cases, noticeably higher friction was observed during Stage 1. For the LST surface, the friction was particularly higher during the first stage of the first cycle, but 
eventually decreased even with decreasing $\lambda$ ratio. This is attributed to lack of the lowfriction tribochemical films at the start of the test, and possibly to fluid film thickness reduction downstream of a dimple [9]. Note that the test started with higher $\lambda$ ratio values. Once the $\lambda$ is low enough and enough wear occurs on the ball to change the contact geometry to a conformal one, significant friction reduction is expected, as observed. Formation of low-friction tribochemical films can also contribute the friction reduction ifformed. Stages 2 to 6 friction was about the same until the extremely low $\lambda$ $(<0.1)$ regime in which the friction was very noisy for polished flat. The generally higher friction observed in test with textured and additive combination is due to the impact of LST on formation and removal of tribochemical films on both the ball and flat surfaces. Figures 4 and 6 show the optical image of the tribofilms from test pair with polished flat and textured flat respectively. Both the ball and flat wear tracks were covered with tribofilm in specimen tested oil containing additives (Figure 4). Figure 5a shows a scanning electron microscope (SEM) micrograph of tribochemical films formed the flat specimen form the additives. Energy dispersive spectroscopy (EDS) analysis of the film shows it contains $M o, S$, and P; key constituents of the additives [Figure 5b]. The figure also contain a peak for Fe from the steel material. In the case of textured surface test pairs, limited amount of tribo film were observed in both flat and ball tracks (Fig. 6). This is consistent with observation that LST do interfere with tribochemical film formation [17]. Streak of film removal can also be seen on the ball wear track (Figure 6a). This may be the result of stress concentration at the rim of the dimples under severe boundary condition resulting in the scraping of the tribo film on the ball surface. This effect also produced more noticeable wear in the ball specimen. 


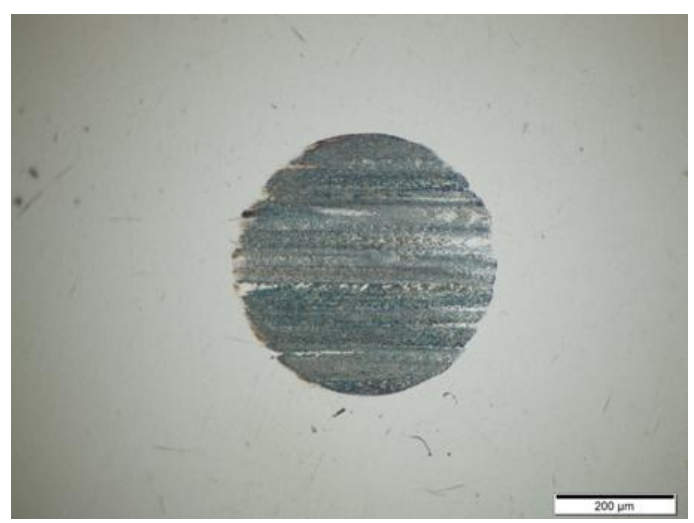

(a)

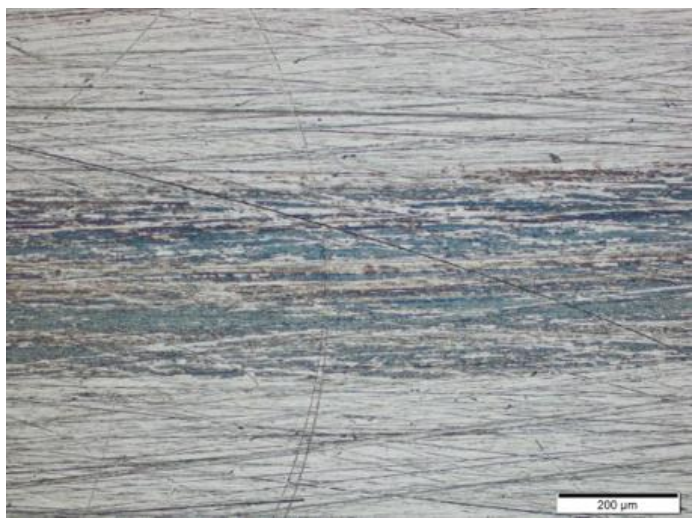

(b)

Figure 4: Optical micrograph of wear track on (a) ball and (b) polished flats tested with additized oil.

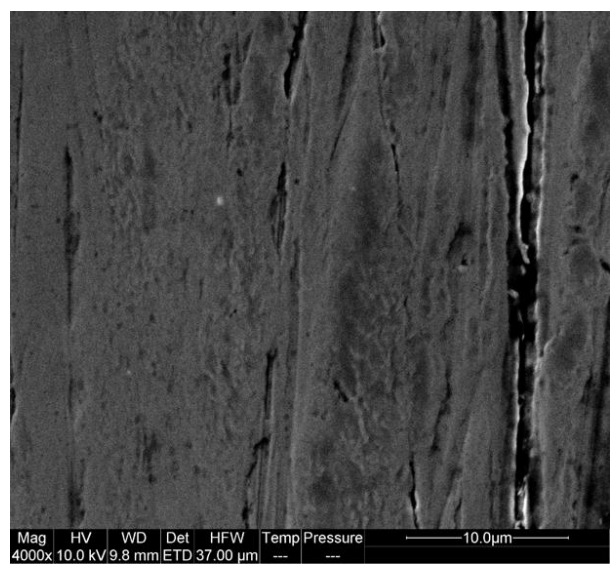

(a)

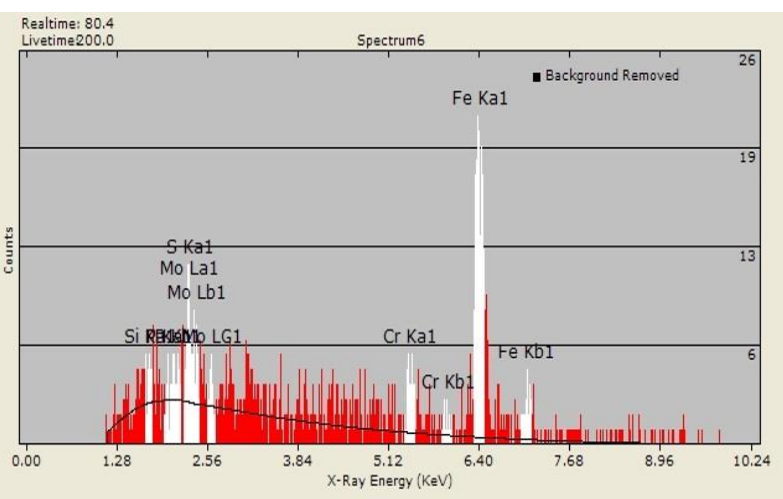

(b)

Figure 5: (a) SEM micrograph of tribochemical surface films on polished flat specimen. (b) EDS analysis of the tribochemical films. 


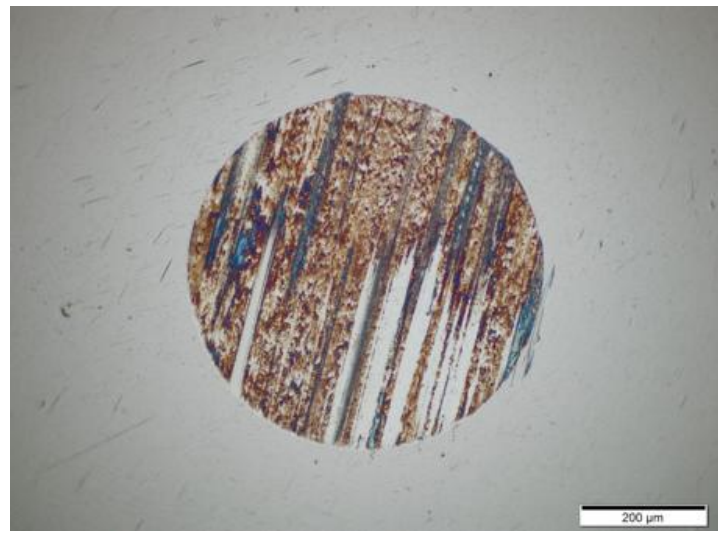

(a)

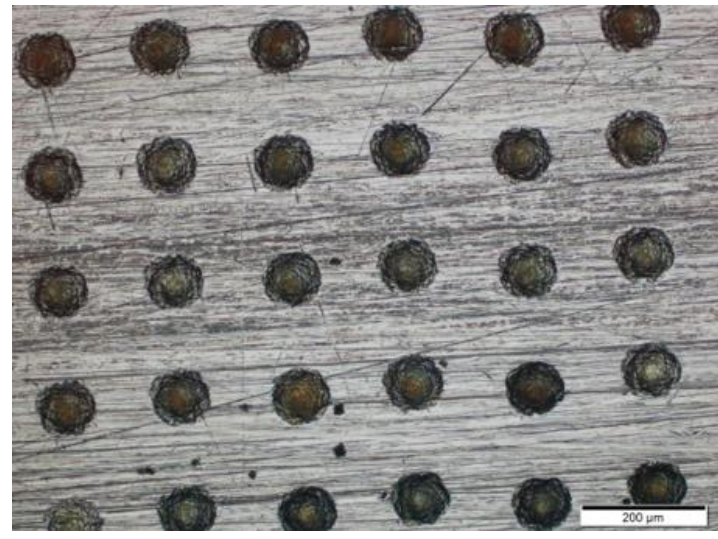

(b)

Figure 6: Optical micrograph of wear track on (a) ball and (b) LST flats tested with additized oil

In terms of wear, non measureable amount of wear or surface damage were observed in all the flat specimens, both polished and textured. Figures $4 \mathrm{~b}$ and $6 \mathrm{~b}$ show typical the wear track on the flat specimens. Measurable amount of wear was however observed in the ball specimens and thus the only basis for wear comparison. Figures $7 \mathrm{a}$ and $7 \mathrm{~b}$ show the optical profilometry of wear track on the ball specimen slid against LST and polished flats respectively. Clearly more wear was observed in the ball sliding against the textured surface compared to sliding on polished surface. This higher ball wear when sliding against LST was observed in tests with both PAO4 base fluid and fluid with additives. Noticeable less wear was observed in tests with oil containing additives as will be expected since an anti-wear additive was included (Figure 8). 

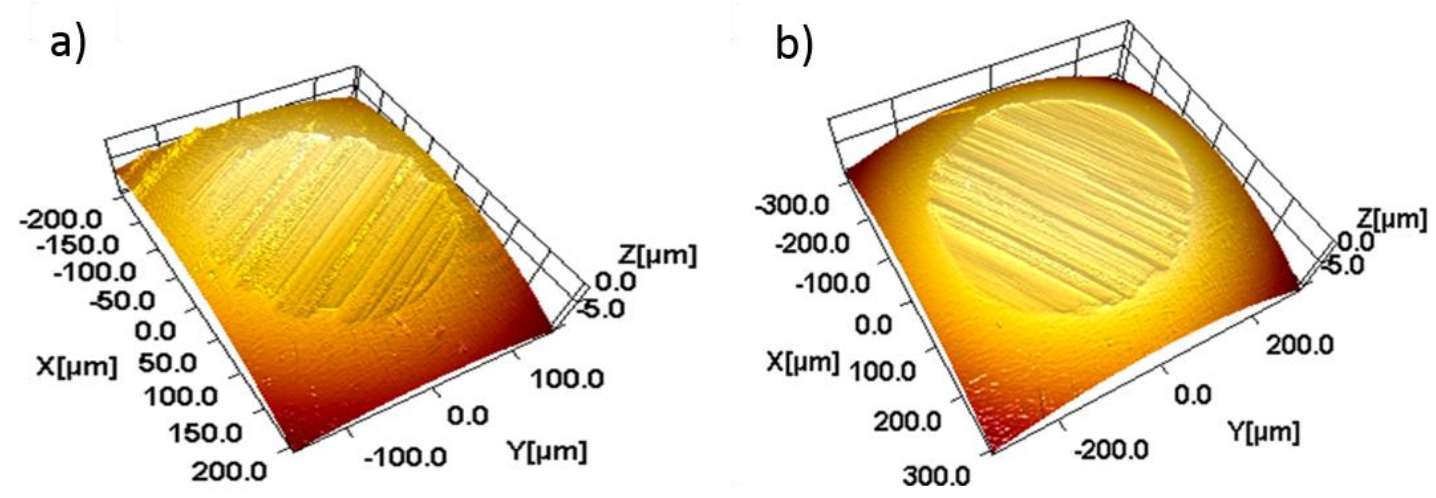

Figure 7: Optical profilometry of wear track on ball tested with lubricant with and without additives sliding against LST flat.

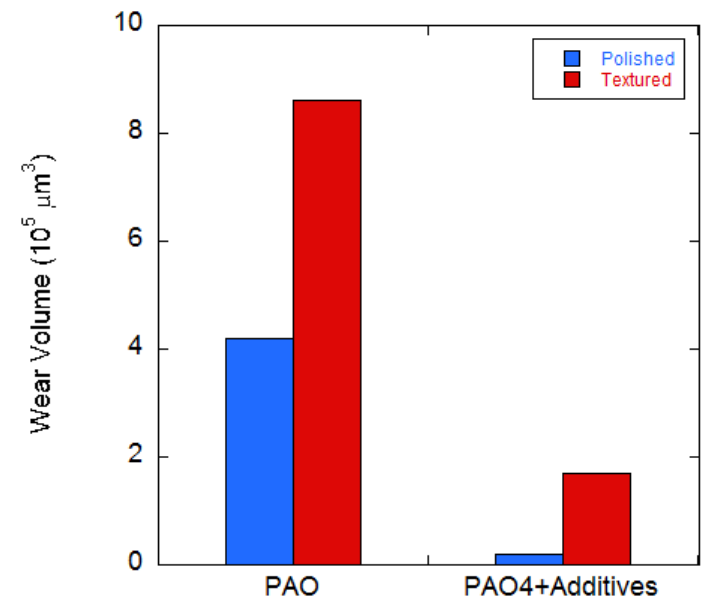

Figure 8: Summary of ball wear in various test conditions.

\section{Conclusion:}

The paper presented the results of evaluation of the combined effects of two friction reduction technologies, namely LST and lubricant additives. The results showed that LST could have a detrimental on the formation of low-friction surface tribochemical surface film from anti-wear and friction modifier additives combination. In the absence of lubricant additives, the LST resulted in friction reduction during unidirectional sliding 
in all lubrication regimes. This is attributed to the effect of fluid film lubrication enhancement by LST. In the presence of low-friction additives however, LST interfered with formation of a stable tribochemical flows, consequently resulting in higher friction than polished surfaces. LST also produced more wear in the sliding ball counterface, with and without oil additives.

\section{Acknowledgement:}

This work was supported by U.S. Department of Energy, Energy Efficiency and Renewable Energy, Office of Vehicle Technologies, under contract DE-AC0206CH11357. The electron microscopy was accomplished at the EMC at Argonne National Laboratory, a U.S. Department of Energy Office of Science Laboratory operated under Contract No. DE-AC02-06CH11357 by UChicago Argonne, LLC.

\section{References:}

1. A. Greco, S. Raphaelson, K. Ehmann and Q. Wang, "Surface Texturing of Tribological Interface Using Vibro-Mechanical Texturing Method," ASME J. Manuf. Sci. Engr., 131 (2009), 1-8.

2. U. Petterson and S. Jacobson," Influence of Surface Texture on Boundary Lubricated Sliding Contacts,” Tribolol. Int., 36 (2003), 857-864.

3. X. Wang, K. Kato, and K. Adachi," The Lubrication Effect of Micropits on Parallel Sliding Faces of SiC in Water," Tribol. Trans, 45 (2002) 294-301.

4. I. Etsion, "State of the Art in Laser Surface Texturing," ASME J. Tribol., 127, (2005), 248-253. 
5. I. Etsion, Y. Kligerman and S. Halperin, "Analytical and Experimental Investigation of Laser-Textured Mechanical Seal Faces," Tribol., Trans, 42 (1999) 511-516.

6. A. Kovalchenko, O. Ajayi, A. Erdemir, G. Fenske, and I. Etsion," The Effect of Laser Surface Texturing on Transition in Lubrication Regimes during Unidirectional Sliding Contact,” Tribol., Inter. 38, (2005), 219-225.

7. P. J. Blau, J. Qu and L. Riester,'Effects of LST on Microstructure and Frictional Characteristics of $\mathrm{ZrO} 2$ Surfaces under Liquid at Solid Lubrication," Proced. Of World Tribol. Congress (2005); WTC 2005-63197.

8. Q. J. Wang and d. Zhu, "Virtual Texturing: Modeling the Performance of Lubricated Contacts of Engineered Surfaces," ASME J. Tribol. 127 (2005) 722728.

9. I. Krupka, M. Hartl, "The Effect of Surface Texturing on Thin EHD Lubrication Films,” Tribol. Inter. 40 (2007) 1100-1110.

10. A. Kovalchenko, O. Ajayi, A. Erdemir and G. Fenske," Friction and Wear Behavior of LST under Lubricated Initial Point Contact,” Wear 271 (2011) 1719-1725.

11. C. Lorenzo-Martin, O. O. Ajayi, J. K. Lee and A. A. Polycarpou, "Frictional Behavior of Boundary Lubricated Contacts - Effect of Tribochemical Films," Proceeding of IJTC, (2009), IJTC 2009-15098.

12. J. Ye, S. Araki, M. Kano, and Y. Yasuda, "Nanometer Scale Mechanical/Structural Properties of MoDTC and ZDDP Tribofilm and Friction Reduction Mechanism," Jap. Jour. Appl. Phys., 44 (2005) 5358-5361. 
13. K. Yamaguchi, C. Sasaki, R. Tsuboi, M. Atherton, T. Storlacki and S. Sosaki, "Effect of Surface Roughness on Friction Behavior of Steel under Boundary Lubrication,” Proc. J. Mech. E. J. Engr. Tribol. 0, (2014), 1-5.

14. A. Morina and A. Neville, "Tribofilms: "Aspect of formation, Stability and Removal,” J. Phys. D: Appl. Phys. 40 (2007), 5476-5487.

15. A. D. Berman, W. A. Ducker, and J. N. Israelachvili, "Origin and Characterization of Different Stick-Slip Friction Mechanisms” Langmuir, 12, (1996), 4559-4563.

16. G. Luengo, J. Israelachvili, and S. Granick, "Generalized Effects in Confined Fluids: New friction map for boundary lubrication” Wear, 200 (1996), 328-335.

17. M. Wakuda, Y. Yamandri, S. Kanzaki and Y. Yasuda, "Effect of Surface Texturing on Friction Reduction between Ceramic and Steel Materials under Lubricated Sliding Contact,” Wear, 254 (2003), 356-363.

18. A. Kovalchenko, O. Ajayi, A. Erdemir, G. Fenske, and I. Etsion, "Effect of LST of steel surface and speed-load parameters on the transition of lubrication regime from boundary to hydrodynamic" STLE Tribo. Trans., 47 (2004), 299-307.

19. O. O. Ajayi, R. A. Erck, C. Lorenzo-Martin, and G. R. Fenske, "Frictional anisotropy under boundary lubrication: effect of surface texture”, Wear, 267 (2009), 1214-1219.

20. O. O. Ajayi, A. Erdemir, R.H. Lee and F. A. Nichols, "Sliding wear of SiC-TiB 2 ceramics matrix composite” J. Amer. Ceram. Soc., 76 (1993), 511-517. 